\title{
Advances in Gallbladder-Preserving Surgery for Gallbladder Diseases
}

\author{
Garba Seydou Aliou ${ }^{1}$, Gang Zhao ${ }^{1}$, An Hua Huang ${ }^{1}$, Long Yang $\mathrm{Yu}^{1}$, Jing Li Cai ${ }^{1}$, Hai Dong Li ${ }^{1}$, Kan Ding ${ }^{1}$, An An Xu ${ }^{1}$, Kai Zhang ${ }^{1}$, \\ Chuan Qi He ${ }^{1}$, Yong Xu${ }^{1}$, Zhong Xin Zhao ${ }^{1}$, Rui Qi Lu ${ }^{1}$, Cheng Zhang ${ }^{1}$, Kaka Katiella ${ }^{2}$, Boureima Ali Nafissatou ${ }^{3}$, and Hai Hu ${ }^{1, *}$ \\ ${ }^{1}$ Department of Gallbladder Diseases Center Affiliated Shanghai East Hospital of Tongji, University School of Medicine, People's Republic of China \\ ${ }^{2}$ Umanand Prasad School of Medicine, Department of Basic Science, University of Fiji, Fiji Islands \\ ${ }^{3}$ Department of Obstetrics and Gynecology, Affiliated to Niamey Hospital of Abdou Moumouni, University School of Medicine, Niamey, Republic of Niger
}

${ }^{*}$ Corresponding author: Hai Hu, Department of Gallbladder Diseases Center Affiliated to Shanghai East Hospital of Tongji, University School of Medicine, People's Republic of China, E-mail: haihulc@hotmail.com

Received: 06 May, 2019 | Accepted: 17 Jun, 2019 | Published: 26 Jun, 2019

Citation: Aliou GS, Zhao G, Huang AH, Yu LY, Cai JL, et al. (2019) Advances in Gallbladder-Preserving Surgery for Gallbladder Diseases. Clin Res Open Access 5(1): dx.doi.org/10.16966/2469-6714.145

Copyright: ( 2019 Aliou GS, et al. This is an open-access article distributed under the terms of the Creative Commons Attribution License, which permits unrestricted use, distribution, and reproduction in any medium, provided the original author and source are credited.

\section{Abstract}

Research on gallbladder diseases, whether in basic pathogenesis or clinical treatment, has been continuing and developing. Currently, gallbladderpreserving lithotripsy has attracted considerable attention in the treatment of cholecystolithiasis in China. In recent years, Endoscopic Minimally Invasive Gallbladder-Preserving Surgery (CGPS) has become more and more mature. However, the recurrence rate of gallstones after CGPS has been an urgent question that requires careful investigation. This article reviews the progress in postoperative recurrence of gallbladder diseases treated with gall bladder preservation surgery.

Keywords: Gallbladder disease; Gallbladder preservation surgery; Treatment; Recurrence; Research progress

\section{Introduction}

Cholecystolithiasis is a common surgical disease with a high incidence in the population, $12 \%$ in Europe, $13.3 \%$ - $17.9 \%$ in the USA and $5.6 \%$ in China. Current research reported that $70 \%$ of gallbladder cancer patients are related to gallstone and this transition can last 1015 years. Gallbladder cancer with gallstone is 13.7 times that without gallstone, indicating that the occurrence of gallbladder cancer is gallstone long-term physical stimulation plus chronic inflammation of mucosa, and bacterial infection [1]. The harmfulness of gallstones is mainly related to the size and number of gallstones. It is not that the smaller the gallstones, the better, the smaller the gallstones will have blocking effect on the gallbladder duct of patients [2]. Pain is strong, at the same time; it will stimulate the patient's gallbladder fluid secretion, resulting in increased internal pressure, and prone to bacterial infection. The main clinical symptoms of cholelithiasis are fever, abdominal pain, and jaundice, which seriously affect patient's livelihood. Cholecystolithiasis is the main treatment in clinical medicine. However, it is particularly important to take some preventive measures to reduce the recurrence rate after CGPC [3].

In this article, we review the literature related to the recurrence of cholecystolithiasis after cholecystolithotomy.

\section{Bile-Conserving Surgery for Asymptomatic Patients with Chronic Cholecystitis and Calculi}

Since Lengenbuch (1882) first used cholecystectomy to treat cholecystolithiasis, it has become the standard treatment mode for cholecystitis and cholecystolithiasis because of its satisfactory therapeutic effect. Laparoscopic Cholecystectomy (LC), first reported by Mouret, 37 years ago, has become the gold standard for the treatment of gallstones due to its advantages of minimal trauma and rapid recovery [3]. However, LC is, in essence, technological innovation of cholecystectomy, including robotic surgery, rather than a change of therapeutic principles [4]. In the face of controversy, many patients and doctors feel confused. With the increasingly strong desire of "removing lesions while preserving gallbladder" as well as the advent of new technology and equipment, whether the consensusbased treatment principles for chronic cholecystitis gallstones should be modified today has become a key issue for research [5]. For gallbladder disease, a common and frequently-occurring disease with high incidence in China, the risk of recurrence after cholecystectomy has attracted much attention. Applying LC to treat cholecystolithiasis seems really easy, but this "easy" is a huge danger. Because of its "easy" and casual implementation of surgical complications, biliary reoperation has become the most abdominal surgery re-operation [6]. By a seemingly simple anatomy operation, patients are not allowed to carry out liver transplantation, disability, or even death, and other adverse consequences occur from time to time. Therefore, many experts and scholars call for careful cholecystectomy, especially for asymptomatic patients [7].

Preventive cholecystectomy can avoid complications such as cholecystitis and pancreatitis secondary to gallstones, but at the same time increases the risk of short-term and long-term complications related to cholecystectomy for patients [8]. However, in the domestic 
literature on gallbladder-conserving lithotripsy, we have observed the random implementation of gallbladder-conserving lithotripsy [9]. Many reports have listed "asymptomatic gallstones" as surgical indications. Documents reported that the indications of operation were "gallstones in all kinds of situations," fullness of upper abdomen only after meals, "discovery of physical examination for units," "minimum age of 4 years and 3 months" [10]. Although 317 papers have been published, and 32090 cases have been reported as of March 2014, which is 5.87 times (317/54) and 7.20 times (32090/4454) of the reported data of Wang Huiqun and others four years ago, respectively, in 2010, there are still many doubts about the exact follow-up results of the recurrence rate of stones from 317 literature analyses [11]. It can be seen from this that many doctors are not yet aware of the exact effect of the operation, because they think that the technology is simple and feasible, they have been carried out at will. In the view of these surgeons, because the technology of gallbladder preservation and stone removal is easy to master, it can be implemented at will [12]. As long as there are stones, they are indications.

Even if they recur later, short-term solutions to some problems can be achieved [13]. When a surgeon considers the treatment strategy of a disease, the first thing to consider is whether the patient needs to use the invasive treatment of "surgery" to solve the problem [14]. Secondly, we should consider what kind of surgical plan to choose to achieve the best therapeutic effect with the least trauma and the least funds [15]. Although gallbladder lithotripsy preserves the gallbladder, it still belongs to invasive surgical technology; patients also bear the risk of anesthesia accident, bile leakage, gastrointestinal tract injury and other surgical complications [16]. If the stone recurs, they should also bear the risk of the second operation and the burden of health economics [17].

It should be clear that asymptomatic patients who can be observed for a long time should not be blindly operated on to remove gallbladder; similarly, it should not be free to preserve gallbladder and stone [18]. Cholecystectomy or cholecystolithotomy should be carefully selected, and excessive treatment should be vigilant $[19,20]$. As for whether long-term observation of asymptomatic gallstones will lead to delayed treatment of gallbladder carcinogenic, this is another aspect of the problem [21]. Our observation of gallstones depends not only on whether there are clinical symptoms but also on taking a variety of biochemical and imaging diagnostic measures to dynamically observe regularly and comprehensively the evolution of its course and to make timely decisions on whether surgery is necessary. This is not inconsistent with the principle of "careful surgery for benign diseases" [22].

\section{Cholecystolithotripsy for Patients with Symptomatic Chronic Cholecystitis and Cholecystolithiasis}

Cholecystolithotomy is one of the treatments for symptomatic chronic cholecystitis and cholecystolithiasis, but its indication deserves our serious consideration [23]. In fact, gallstone preservation and extraction are not innovative technology. In fact, it has been tried for many years [24]. In the same period of discovering the harm of cholecystitis and cholecystolithiasis to human body, cholecystectomy is almost used to treat the disease. Some scholars have been discussing the possibility of removing and retaining gallstones by means of drug litholysis, lithotripsy, and extracorporeal shock wave lithotripsy, just because of a large number of randomized controlled studies. The treatment effect was not good and was gradually eliminated naturally [25]. The emergence of endoscopic gallbladder-preserving lithotripsy has brought good news to satisfy patient's good wish of "eliminating disease and preserving gallbladder" [26]. However, it is still a technical improvement of applying high-tech means to treat chronic cholecystitis and cholecystolithiasis, and it has not solved the mechanism of stone formation and the fundamental problem of recurrence in the future [27]. This method is mainly used in the emergency treatment of highrisk patients in foreign countries, and then selective cholecystectomy to remove lesions so that the disease can be fundamentally controlled, while many domestic scholars intend to take gallbladder preservation as a treatment alternative to LC [28]. According to the literature analysis, the feasibility of the following situations still needs to be considered carefully.

Also, at present, there is no sufficient and reliable evidence to confirm that histological changes of gallbladder mucosa with significant inflammatory and proliferative histological changes can be completely normal due to the removal of stones [29]. However, a large number of studies have confirmed that chronic inflammation of gallbladder tissue may lead to atypical hyperplasia of mucosal epithelium, longterm compression of gallbladder mucosa by stones larger than $3 \mathrm{~cm}$ in diameter and other factors may lead to carcinogenesis [30].

In China, with gallstones $>3.6 \mathrm{~cm}$, atrophic cholecystitis, gallstones filled gallbladder (up to 823 stones, and mucosal tissue with no histopathological changes of atypical hyperplasia), yet gallstone preservation is adopted [31].

The relationship between cholelithiasis and metabolism, family history and genetic inheritance [32]. The joint study of Ruijin Hospital Affiliated to Shanghai Jiaotong University and Karolinska Institute in Sweden shows that cholesterol gallstone disease belongs to the category of metabolic syndrome. Abnormal lipid metabolism in intestinal and hepatic axis, including hepatobiliary and small intestinal systems, is an important component of the pathogenesis of gallstone [33]. Its characteristics are genes related to lipid metabolism (such as cholesterol transporter-3 in the lateral membranes of the bile duct). Adenosine Phosphate Binding Cassette (ABC) G5/G8, liver X-receptor alpha (LXR alpha) and Scavenger Receptor-B Type-I (SRB1) were abnormally increased [34]. However, in many clinical studies of gallstone preservation and extraction, there is no detailed information about the family history of patients with gallstones, the detection of biochemical indicators such as blood lipid and cholesterol metabolism, and the abnormality of metabolic function [35]. Some scholars who advocate cholecystolithiasis preservation believe that the formation of gallbladder stones has nothing to do with gallbladder [36]. The occurrence of gallbladder stones is due to abnormal cholesterol metabolism. Therefore, only removing the gallstones from the gallbladder does not solve the metabolic and other etiological problems. Thus these cases are not suitable for cholecystectomy [37]. Other studies have shown that the formation of gallstones in the gallbladder is closely related to decrease Cholecystokinin (CCK) receptors, and decrease in signal transduction, which are important gastrointestinal hormones regulating gallbladder motility [38]. Consequently, the gallbladder becomes the final organ of gallstone disease.

The standardization of gallbladder function assessment before operation [39]. Preoperative assessment of gallbladder function is an important basis for the selection of treatment strategies for chronic cholecystitis and gallstones [40]. Most of the literature reports on gallbladder preservation and lithotripsy listed "good gallbladder function" and "B-mode ultrasonography examination of gallbladder contraction function $(>30 \% \sim 40 \%)$ as indications [41]. There were also no clear methods for gallbladder function assessment and no objective evaluation criteria for homogeneity. It was difficult to assess the reliability and authenticity of "normal gallbladder function" 
described by various authors because of different methods and sensory estimation. Currently, there are three main methods for evaluating gallbladder contractile function [42]:

(i) One is oral cholecystography, which has been basically abandoned.

(ii) $99 \mathrm{Te}$, ETC scans requiring special equipment is difficult to popularize in most primary hospitals [43];

(iii) B-mode ultrasonography is the most commonly used method in hospitals.

It should be clearly pointed out that the criteria for determining gallbladder contraction function determined by Ultrasound are:

(1) Gallbladder contraction function is good: within 2 Hours after meal, gallbladder emptying or contraction $>2 / 3$ is normal [44].

(2) The gallbladder contraction function is poor: the gallbladder contraction $<1 / 2$ within 2 Hours after meal is suspicious.

(3) The function of gallbladder contraction is poor: the gallbladder contraction $<1 / 3$ within 2 Hours after meal is abnormal.

(4) The gallbladder has no contractile function: 2 Hours after meal, the gallbladder is the same size as the empty stomach [45].

If the empty stomach gallbladder is smaller than the normal size, it often indicates severe lesions and loss of function. If the gallbladder is enlarged, it means that there is obstruction below the gallbladder. In the improved B-mode ultrasonography, a 3D gallbladder function detection and judgment criteria introduced by Jiang ZY, et al. [46] and others also pointed out that the normal gallbladder function criteria are gallbladder contraction rate $(>75 \%)$ and gallbladder wall thickness $(<3 \mathrm{~mm})$. The reduction of gallbladder contraction rate or the thickening of the gallbladder wall, either of which is not within the normal range, means that gallbladder function is abnormal [47]. In the domestic literature, it is inaccurate for many authors to define gallbladder contraction $(>30 \%)$ and gallbladder wall thickness $(<6$ $\mathrm{mm}$ ) as the criteria of "good function" [48] rather it should belong to the category of "poor gallbladder function." It is not rigorous and correct to incorporate the chronic cholecystitis gallstones with poor gallbladder function into the indication category of "gallstone preservation and extraction" [49].

It is particularly worrying whether Occult cystic duct cancer is difficult to early diagnosis and treatment. The prognosis of gallbladder cancer itself is very poor, because its pathological characteristics are adenocarcinoma accounting for $89.4 \%$ of the incidence, of which the relatively well-differentiated papillary adenocarcinoma accounts for only 5.7\% [50]. Most of them are poorly differentiated adenocarcinoma and signet ring cell carcinoma with poor prognosis. One of the important updates of American Joint Committee on Cancer (AJCC) version 7 is to plan cystic duct cancer as gallbladder cancer [51]. Pericystic nerve invasion, lymph node metastasis and vascular invasion in cystic duct cancer were significantly higher than those in other types of cystic duct cancer, while the 3-year and 5 -year survival period was significantly reduced. Statistical analysis showed that the prognosis of cystic duct cancer was relatively poor if it invaded the cystic duct. The $7^{\text {th }}$ edition of AJCC divides the lymph node metastasis of gallbladder cancer into two stations: N1 and N2. $\mathrm{N} 1$ is the lymph node of porta hepatis while lymph nodes of celiac trunk, paraduodenal, peripancreatic and superior mesenteric artery are considered distant metastasis $(\mathrm{N} 2)$. N1 and N2 were used as markers of stage IIIB and IVB respectively. Shirai Y, et al. [52] has injected dyes into the lymphatic vessels of the gallbladder to show the way of lymphatic drainage of the gallbladder. It was found that the lymphatic drainage around the common bile duct at N1 station could directly converge to the lymph nodes around the abdominal aorta and inferior vena cava at M1 station. As a result, the spread and metastasis of the whole body were rapidly induced, and the course of the disease turned to stage IIIB and IVB. The narrow gallbladder neck duct is the site where stones are easy to stay, rub and incarcerate. The mucosal epithelium is most likely to be damaged, and the occurrence of tumors is more likely.

Post-operatively, special attention should be paid to the pathological observation of the neck of gallbladder for early detection and radical remedial operation. The objective results of pathological examination cannot be obtained after gallbladder preservation and stone removal in patients with gallbladder neck duct incarceration will lose the opportunity of early detection and timely treatment. There is need to confirm whether the gallbladder duct is unobstructed after operation and for presence of "white bile in the gallbladder." In the case of incarcerated gallstones in the neck of the gallbladder, whether bile can be seen flowing out of the gallbladder or not after removal of the gallstones, biliary-conserving surgery should be contraindicated to prevent missed diagnosis of gallbladder-neck duct cancer, which is a matter of principle in the choice of treatment.

\section{Dispute Over Decision-Making in the Treatment of Gallbladder Polyps}

In 1991, Wang divided preoperative polypoid gallbladder detected by B-mode ultrasonography into three categories according to the pathological results of 100 cases of surgical treatment of polypoid gallbladder [53]. In view of the current technical level, the pathological nature of gallbladder polyps is difficult to judge prior to operation, so we should be careful to use gallbladder-preserving therapy.

\section{Standardization of Postoperative Management}

Studies have shown that traditional Chinese medicine with antiinflammatory and cholagogic effects has been widely used in the treatment of cholecystolithiasis and chronic cholecystitis. The effect of Danning tablet on CGPS is as follows: relaxing the sphincter of biliary tract, preventing colic and bile leakage; speeding up the disappearance of traumatic inflammation of gallbladder after operation and promoting the recovery of gallbladder function can prevent and change the tendency of cholesterol stone formation in bile. Animal experiments have proved that Danning tablet can significantly reduce the activity of beta-elucidation in liver and bile, reduce the content of free bilirubin and calcium ion in bile, thus reversing the trend of gallstone formation, and has the effect of preventing gallstone formation [52]. Therefore, the drug has the dual effect of preventing cholesterol and cholecystolithiasis formation [54]. The Zhongshan Hospital affiliated to Fudan University has followed up 792 patients whose stones have disappeared after conservative treatment. The results showed that the recurrence rate of stones in 1,2,3,4,5 years and beyond were $11.6 \%$, $22.3 \%, 24.5 \%, 36.4 \%, 39.3 \%$ and $39.6 \%$, respectively [55,56]. From 67 articles with recurrence rate out of 317 researches $(21.1 \%)$ reported recurrence rate ranging from $2.8 \%$ to $36.5 \%$, where only $446 / 6519$ patients follow-up (i.e. $82.1 \%$ [55/67 cases], follow-up $<5$ years) [57]. Is the effect of gallstone-conserving lithotripsy really improved or is the follow-up investigation not meticulous enough for diagnosis? or the important prognostic indicator was missed! $59.0 \%$ (i.e., $187 / 317$ reports) did not follow up the recurrence rate, and $19.9 \%(63 / 317)$ reported that the recurrence rate was 0 , follow-up time $<4$ years [58]. Most of the literature did not have statistical analysis data on the correlation between follow-up time 
and recurrence rate of stones in the corresponding period. Some simply reported the follow-up time of " 1 month to 3 years", and only one article used life table method to calculate recurrence rate after operation [59]. The overall real recurrence rate of stone is difficult to estimate.

Nonetheless, these results suggest that gallstone-conserving lithotripsy has been carried out vigorously in China. The existing literature shows that the scientific nature of relevant research is insufficient. There is no definite high-level report on how to prevent the recurrence of gallstones after gallstone-conserving surgery [60]. The exact effect of ursodeoxycholic acid or other methods to prevent stone recurrence remains to be confirmed. At present, the formulation of its normative measures is still uncertain, which is an important issue to be further studied. If ursodeoxycholic acid and other drugs can really prevent the recurrence of gallstones, the desire to block the formation of gallstones can also be realized, whether cholecystectomy or gallbladder-preserving surgery may be withdrawn from the historical stage, just as the use of H2-receptor inhibitors has made peptic ulcer a disease that can be cured by basic medical treatment.

\section{Conclusion}

In conclusion, the authors believe that the treatment principles proposed by Academician Huang Zhiqiang are not outdated and should be a consensus concept for us to understand this issue when we are choosing the treatment strategies for patients with chronic cholecystitis and gallstones. Considering the developmental stages of the patient's course and the relevant specific circumstances, and the different treatment methods adopted; the treatment principle should not be easily changed. For asymptomatic cholecystolithiasis, treatment should be carried out mainly under the condition of regular follow-up and appropriate measures that may control the growth of gallstones. Cholecystectomy should be performed resolutely for patients with recurrent cholecystitis, stone diameter $>3 \mathrm{~cm}$, gallbladder wall thickness $>4 \mathrm{~mm}$, filling gallbladder stones, atrophic cholecystitis, gallbladder neck duct stones, and chronic calculous cholecystitis with complications and malignant tendency. Based on the analysis of the current literature and the requirements of evidence-based medicine, the domestic gallstone-conserving surgery lacks standardized indications and technical operating standards, and is still in the exploratory stage of "crossing the river by touching the stones", the exact effect of gallbladder-preserving lithotripsy should be tried in two stages of gallstone disease: (1) to treat patients with mild symptoms (e.g. small number of gallstones, no metabolic syndrome nor family history of gallstones), and strong personal willingness to preserve gallbladder. Stone recurrence should be treated, and preparations for gallbladder resection should be made after recurrence. (2) For the elderly and high-risk population with acute attack of cholecystitis, coexistence of more diseases, intolerance of cholecystectomy, temporary emergency surgery should be performed by decision-making. Academicians Qiu Fazu and Huang Zhiqiang did pay close attention to the problem of gallbladder preservation for gallbladder with good function and no obvious histopathological changes, rather than advocating that all gallbladders with pathological changes should be preserved. We should make great efforts to apply the concept of precise surgery to strengthen the technical management of chronic cholecystitis and cholecystolithiasis to relieve the pain of patients to the greatest extent with the least trauma, the best treatment strategy, and the least economic cost.

\section{References}

1. Muszynska C, Lundgren L, Lindell G, Andersson R, Nilsson J, et al. (2017) Predictors of incidental gallbladder cancer in patients undergoing cholecystectomy for benign gallbladder disease: results from a population-based gallstone surgery registry. Surgery 162: 256-263.

2. Yamaguchi J, Kaneoka Y, Maeda A, Takayama Y, Onoe S, et al. (2016) Benefit of extended radical surgery for incidental gallbladder carcinoma. Surg Today 46: 453-459.

3. Krishnamurthy G, Singh H, Rajendran J, Sharma V, Yadav TD, et al. (2016) Gallbladder tuberculosis camouflaging as gallbladder cancercase series and review focussing on treatment. Ther Adv Infect Dis 3: $152-157$.

4. Chaudhary D, Ahluwalia R, Rai A (2017) Synchronous breast cancer and gallbladder diseases-a chromosomal analysis: a pilot study at a tertiary care centre. Indian J Surg 79: 544-548.

5. Zhang M, Meng T, Cheng F, Liu Y (2016) Clinic study on the association between gallbladder diseases and colorectal cancer. Chinese Journal of Cancer Prevention and Treatment.

6. Zhou D, Wang JD, Yang Y, Yu WL, Zhang YJ, et al. (2016) Individualized nomogram improves diagnostic accuracy of stage I-II gallbladder cancer in chronic cholecystitis patients with gallbladder wall thickening. Hepatobiliary Pancreat Dis Int 15: 180-188.

7. Basak F, Hasbahceci M, Canbak T, Sisik A, Acar A, et al. (2016) Incidental findings during routine pathological evaluation of gallbladder specimens: review of 1,747 elective laparoscopic cholecystectomy cases. Ann R Coll Surg Engl 98: 280-283.

8. Tekcham DS, Poojary SS, Bhunia S, Barbhuiya MA, Gupta S, et al. (2016) Epigenetic regulation of APC in the molecular pathogenesis of gallbladder cancer. Indian J Med Res 143: S82-S90.

9. Ko H, Choi I, Chang K, Jeong G, Gong G, et al. (2016) Amphiphilic metabolites in gallbladder bile: potential biomarkers for gallbladder diseases. Applied Spectroscopy Reviews 51: 706-717.

10. Dixit R, Pandey M, Tripathi SK, Dwivedi AN, Shukla VK (2017) Comparative analysis of mutational profile of sonic hedgehog gene in gallbladder cancer. Dig Dis Sci 62: 708-714.

11. Ott OJ (2018) Multimodality Treatment for Bladder Conservation. Urologic Oncology.

12. Virani NA, Davis C, Mckernan P, Hauser P, Hurst RE, et al. (2018) Phosphatidylserine targeted single-walled carbon nanotubes for photothermal ablation of bladder cancer. Nanotechnology 29: 035101.

13. Cadish LA, Osann KE, Noblett KL (2017) Stimulation latency and comparison of cycling regimens in women using sacral neuromodulation. Neurourol Urodyn 36: 486-489.

14. Yoshida MA, Yamamoto D, Sato K (2017) Physostomous channel catfish, ictalurus punctatus, modify swimming mode and buoyancy based on flow conditions. J Exp Biol 220: 597-606.

15. Kamada Y, Murayama Y, Arita T, Kosuga T, Konishi H, et al. (2016) Intraoperative 5-aminolevulinic acid-mediated photodynamic diagnosis of gallbladder cancer: a case report. Photodiagnosis Photodyn Ther 14: 74-76.

16. Foerster C, Koshiol J, Guerrero AR, Kogan MJ, Ferreccio C (2016) The case for aflatoxins in the causal chain of gallbladder cancer. Med Hypotheses 86: 47-52. 
17. Lee SE, Kim SW, Han HS, Lee WJ, Yoon DS, et al. (2018) Surgical strategy for T2 gallbladder cancer: Nationwide multicenter survey in korea. J Korean Med Sci 33: e186.

18. Goussous N, Hosseini M, Sill AM, Cunningham S (2016) Mo1460 Minimally invasive and open gallbladder cancer resections: 30-vs 90-day mortality. Gastroenterology 150: S1236-S1237.

19. Chirilă DN, Chirilă MD, Turdeanu NA, Dudric VN, Pop TR (2016) The Glutathione S-Transferases (GSTS) gene polymorphisms in hepatocellular, pancreatic and gallbladder cancers. Hvm Bioflux 8: 34-40.

20. Sugimoto M, Takagi T, Suzuki R, Konno N, Asama H, et al. (2017) Contrast-enhanced harmonic endoscopic ultrasonography in gallbladder cancer and pancreatic cancer. Fukushima J Med Sci 63: 39-45.

21. Wang SH, Wu XC, Zhang MD, Weng MZ, Zhou D, et al. (2016) Upregulation of h19 indicates a poor prognosis in gallbladder carcinoma and promotes epithelial-mesenchymal transition. Am J Cancer Res 6: 15-26.

22. Cha BH (2015) Epidemiological characteristics of gallbladder cancer in Jeju Island: a single-center, clinically based, age-sex-matched, case-control study. Asian Pac J Cancer Prev 16: 8451-8454.

23. Tekcham DS, Tiwari PK (2016) Epigenetic regulation in gallbladder cancer: promoter methylation profiling as emergent novel biomarkers. Asia Pac J Clin Oncol 12: 332-348.

24. Cha BH, Lee BS, Lee SH, Kang SJ, Park MJ (2017) A study of alcohol consumption and obesity as main risk factor for symptomatic gallbladder stone: a Case-Control Study. Asian Pac J Cancer Prev 18: 715-719.

25. Tihan D, Kafa IM, Duman U, Erol F, Dilektasli E, et al. (2016) The effect of morphometric and anatomic relationship of gallbladder to the liver on standard laparoscopic cholecystectomies and proposal of a new anatomical classification. Eur Rev Med Pharmacol Sci 20: 1458-1466.

26. Lundgren L, Muszynska C, Ros A, Persson G, Gimm O, et al. (2018) Are Incidental Gallbladder Cancers Missed With A Selective Approach Of Gallbladder Histology At Cholecystectomy? World J Surg 42: 10921099.

27. Nitta T, Kataoka J, Ohota M, Fujii K, Takashima Y, et al. (2018) Surgical strategy for suspected early gallbladder carcinoma including incidental gallbladder carcinoma diagnosed during or after cholecystectomy. Ann Med Surg (Lond) 33: 56-59.

28. Castro Ruiz C, Pedrazzoli C, Bonacini S (2016) Gallbladder's clear cell renal carcinoma metastasis: A case report. Int J Surg Case Rep 18: 48-51.

29. Olthof PB, Metman MJH, de Krijger RR, Scheepers JJ, Roos D, et al. (2018) Routine Pathology and Postoperative Follow-Up are Not Cost-Effective in Cholecystectomy for Benign Gallbladder Disease. World J Surg 42: 3165-3170.

30. Chantarojanasiri T, Hirooka Y, Kawashima H, Ohno E, Kongkam P, et al. (2017) The role of endoscopic ultrasound in the diagnosis of gallbladder diseases. J Med Ultrason (2001) 44: 63-70.

31. Thiels CA, Hanson KT, Chawla KS, Topazian MD, Paley KH, et al. (2016) Functional gallbladder disease: operative trends and shortterm outcomes. Surgery 160: 100-105.

32. Grochola LF, Soll C, Zehnder A, Wyss R, Herzog P, et al. (2017) Robotassisted single-site compared with laparoscopic single-incision cholecystectomy for benign gallbladder disease: protocol for a randomized controlled trial. BMC Surg 17: 13.
33. Dakour Aridi H, Sultanem S, Abtar H, Safadi BY, Fawal H, et al. (2016) Management of gallbladder disease after sleeve gastrectomy in a selected lebanese population. Surg Obes Relat Dis 12: 1300-1304.

34. CholeS Study Group, West Midlands Research Collaborative (2016) Population-based cohort study of outcomes following cholecystectomy for benign gallbladder diseases. Br J Surg 103: 1704-1715.

35. Li M, Wu, H, Wei S (2016) New-Style Laparoscope and Endoscope Cooperative Gallbladder-Preserving Surgery for Polyps. J Coll Physicians Surg Pak 26: 91-95.

36. Otte CM, Penning LC, Rothuizen J (2017) Feline biliary tree and gallbladder disease: Aetiology, diagnosis and treatment. J Feline Med Surg 19: 514-528.

37. Bonatti M, Vezzali N, Lombardo F, Ferro F, Zamboni G, et al. (2017) Gallbladder adenomyomatosis: imaging findings, tricks and pitfalls. Insights Imaging 8: 243-253.

38. Han C, Shan X, Yao L, Yan P, Li M, et al. (2018) Robotic-assisted versus laparoscopic cholecystectomy for benign gallbladder diseases: a systematic review and meta-analysis. Surg Endosc 32: 4377-4392.

39. Teng SF, Xin-Xing LI, Zhang YY, Zhang, WG, Zhang XW, et al (2017) Clinical value of tro $\mathrm{p} 2$, pten and $\mathrm{p}$-akt expression in gallbladder carcinoma. Journal of Hepatopancreatobiliary Surgery.

40. Mizutani S, Torisu, S, Kaneko Y, Yamamoto S, Fujimoto S, et al. (2017) Retrospective analysis of canine gallbladder contents in biliary sludge and gallbladder mucoceles. J Vet Med Sci 79: 366-374.

41. Patel K, Dajani K, lype S, Chatzizacharias NA, Vickramarajah S, et al. (2016) Incidental non-benign gallbladder histopathology after cholecystectomy in an united kingdom population: need for routine histological analysis? World J Gastrointest Surg 8: 685-692.

42. Al-Hakkak SMM (2017) Agenesis of gall bladder in laparoscopic cholecystectomy-A case report. Int J Surg Case Rep 39: 39-42.

43. Lian J, Ma Y, Ma Y, Shi B, Liu J, et al. (2017) Automatic gallbladder and gallstone regions segmentation in ultrasound image. Int J Comput Assist Radiol Surg 12: 553-568.

44. Negrão de Figueiredo G, Mueller-Peltzer K, Zengel P, Armbruster $M$, Rübenthaler J, et al. (2018) Diagnostic performance of contrastenhanced ultrasound (CEUS) for the evaluation of gallbladder diseases1. Clin Hemorheol Microcirc 69: 83-91.

45. Sharafinski ME Jr, Meyers AB, Vorona G (2017) Pediatric cholangiopathies: diseases of the gallbladder and biliary tract. Abdom Radiol (NY) 42: 69-85.

46. Jiang ZY, Sheng X, Xu CY, Li WW, Chang XX, et al. (2013) Gallbladder gallstone disease is associated with newly diagnosed coronary artery atherosclerotic disease: a cross-sectional study. PLoS One 8: e75400.

47. Haghighatafshar M, Amirkhani Z, Ghaedian T (2018) Role of Hepatobiliary Scintigraphy in the Diagnosis of Gallbladder Agenesis; A Case Report And Brief Review of Literature. Middle East J Dig Dis 10: $109-113$.

48. Saafan T, Hu JY, Mahfouz AE, Abdelaal A (2018) True left-sided gallbladder: a case report and comparison with the literature for the different techniques of laparoscopic cholecystectomy for such anomalies. Int J Surg Case Rep 42: 280-286.

49. Quoraishi S, Ahmed J, Ponsford A, Rasheed A (2017) Lessons learnt from a case of extracorporeal shockwave lithotripsy for a residual gallbladder stone. Int J Surg Case Rep 32: 43-46. 
50. Lee H, Kim K, Park I, Cho H, Gwak G, et al. (2016) Preoperative predictive factors for gallbladder cholesterol polyp diagnosed after laparoscopic cholecystectomy for polypoid lesions of gallbladder. Ann Hepatobiliary Pancreat Surg 20: 180-186.

51. Musleh, MG, Burnett H, Rajashanker B, Ammori BJ (2017) Laparoscopic double cholecystectomy for duplicated gallbladder: a case report. Int J Surg Case Rep 41: 502-504.

52. Shirai Y, Yoshida K, Tsukada K, Ohtani T, Muto T (1992) Identification of the regional lymphatic system of the gallbladder by vital staining. Br J Surg 79: 659-662.

53. Liska V, Treska V, Skalicky T, Fichtl J, Bruha J, et al. (2017) Evaluation of tumor markers and their impact on prognosis in gallbladder, bile duct and cholangiocellular carcinomas-a pilot study. Anticancer Res 37: 2003-2009.

54. Lau CSM, Zywot A, Mahendraraj K, Chamberlain RS (2017) Gallbladder carcinoma in the United States: A Population Based Clinical Outcomes Study Involving 22,343 Patients from the Surveillance, Epidemiology, and End Result Database (1973-2013). HPB Surg 2017: 1532835.

55. Akateh C, Arnold CA, Benissan-Messan D, Michaels A, Black SM (2018) Cystoisospora belli Gallbladder Infection in a Liver Transplant Donor. Case Report Infect Dis 2018: 3170238.
56. Mathis R, Stodghill J, Shaver T, Younan G (2017) Cholecystectomy of an Intrahepatic Gallbladder in an Ectopic Pelvic Liver: A Case Report and Review of the Literature. Case Rep Surg 2017: 3568768.

57. Okada K, Kawai M, Ueno M, Hirono S, Miyazawa M, et al. (2016) Depth of Hepatic Infiltration and Lymph Node Swelling as factors for Considering Surgery for T2-4 Gallbladder Carcinoma Patients. Anticancer Res 36: 3075-3080.

58. Jain D, Bhandari BS, Agrawal N, Singhal S (2018) Endoscopic Ultrasound-Guided Gallbladder Drainage Using a Lumen-Apposing Metal Stent for Acute Cholecystitis: A Systematic Review. Clin Endosc 51: 450-462.

59. Sunada F, Morimoto N, Tsukui M, Kurata H (2017) The rendezvous technique involving insertion of a guidewire in a percutaneous transhepatic gallbladder drainage tube for biliary access in a case of difficult biliary cannulation. J Rural Med 12: 46-49.

60. Kim MH, Yeo SJ, Jung MK, Cho CM (2016) The Impact of Gallbladder Status on Biliary Complications After the Endoscopic Removal of Choledocholithiasis. Dig Dis Sci 61: 1165-1171. 